Tropical Journal of Pharmaceutical Research February 2011; 10 (1): 61-67

(C) Pharmacotherapy Group,

Faculty of Pharmacy, University of Benin,

Benin City, 300001 Nigeria.

All rights reserved.

Research Article

Available online at http://www.tjpr.org

\title{
Rifabutin-loaded Floating Gellan Gum Beads: Effect of Calcium and Polymer Concentration on Incorporation Efficiency and Drug Release
}

\author{
Anurag Verma ${ }^{1 *}$ and Jayant K Pandit ${ }^{2}$ \\ ${ }^{1}$ Department of Pharmaceutics, College of Pharmacy, IFTM, Moradabad, 244001, ${ }^{2}$ Department of Pharmaceutics, \\ Institute of Technology, Banaras Hindu University, Varanasi, India
}

\begin{abstract}
Purpose: To formulate rifabutin-loaded floating gel beads for stomach-specific release.

Methods: Rifabutin-loaded floating gellan gum beads were prepared by ionotropic calcium-induced gelation in acidic medium. In-vitro buoyancy and drug release studies were performed using a USP dissolution apparatus type II in $0.01 \mathrm{M} \mathrm{HCl}$ (ph 2.0) as the dissolution medium. The shape, surface morphology and internal structure of the dried beads were examined by scanning electron microscopy. Fourier Transform infrared spectroscopy (FTIR) was applied to investigate drug-polymer interactions.

Results: The beads exhibited excellent buoyancy in simulated gastric fluid (SGF) and remained buoyant for $18 \mathrm{~h}$. Drug incorporation efficiency of the beads ranged from 40 to $60 \%$ and was dependent significantly $(p<0.05)$ on the concentrations of calcium ions and gellan gum. Drug release from the floating bead formulations was rapid, with $>50 \%$ of the drug released within $1 \mathrm{~h}$. Increased polymer concentration did not significantly $(p<0.05)$ retard drug release.

Conclusion: Incorporation efficiency and release of rifabutin can be controlled by modulation of the investigated parameters. The developed floating gellan gum beads may be suitable for a potential oral stomach-specific release system to treat stomach infections such as multi-drug resistant Helicobacter pylori infection.
\end{abstract}

Keywords: Floating beads, Gellan gum, Incorporation efficiency, Rifabutin, Stomach-specific release. 


\section{INTRODUCTION}

Gellan gum is a bacterial exopolysaccharide commercially prepared by aerobic submerged fermentation of Sphingomonas elodea. There are two chemical forms of gellan gum: native or natural form, which has high acyl contents, and low or deacetylated form [1]. In the presence of counterions, this polymer is capable of forming gels that are particularly strong when formed with divalent ions. The degree of acylation also influences the strength of the resulting network. Indeed, in its native form, gellan is acylated and it forms soft, elastic, transparent and flexible gels while de-acylation leads to hard, nonelastic and brittle gels [2].

The gelling mechanism of gellan can be induced by cations and is temperaturedependent. In aqueous solution, the gelation of gellan is accompanied by a two-step process, namely, formation of double helices from random coil chains and an aggregation of pairs of double helices[3]. The coil-helix transition is greatly affected by electrostatic interaction with cations present in the solution. Gellan forms gels in the presence of monovalent $\left(\mathrm{Na}^{+}, \mathrm{K}^{+}\right)$and divalent cations $\left(\mathrm{Ca}^{2+}, \mathrm{Mg}^{2+}\right)$ but its affinity for the latter is much stronger than for the former [1]. A number of efforts have been made to understand the mechanism of microencapsulation of gellan gum in the presence of calcium cations but the exact mechanism remains unknown $[2,3]$.

The objective of this study was to evaluate the effect of calcium ions on the encapsulation efficiency of deacylated gellan gum incorporating rifabutin as a model drug.

\section{EXPERIMENTAL}

\section{Materials}

Rifabutin was obtained from Henan Kangtai Pharm Group, China. Deacetylated gellan gum (Kelcogel) was obtained as a gift from CP Kelco, UK Ltd, Surrey, U.K. De-ionized water (HPLC grade) purchased from Quilagens, India was used. All other chemicals used were of AR grade.

\section{Preparation of floating beads}

The beads, whose composition is shown in Table 1, were prepared by ionotropic gelation technique [4] with some modification. Gellan solution $(1-3 \% \mathrm{w} / \mathrm{v})$ was prepared by dissolving gellan in deionized water at $90{ }^{\circ} \mathrm{C}$. The drug, rifabutin, and sodium bicarbonate were dissolved/dispersed uniformly in the gellan solution at or just below $40^{\circ} \mathrm{C}$ with continuous stirring until a uniform dispersion was obtained. The bubble-free slurry (dispersion) was added drop-wise into the gelation medium consisting of varying concentrations of $\mathrm{Ca}^{2+}$ in $10 \% \mathrm{v} / \mathrm{v}$ acetic acid with a $25 \mathrm{ml}$ hypodermic syringe through a 20 $G$ needle into the gelation medium. The medium was continuously stirred during bead formation to enhance the mechanical strength of the beads and also to prevent their aggregation. The beads were cured for 5 min, separated by filtration, washed thrice with deionized water and dried at $40{ }^{\circ} \mathrm{C}$ in a hot air oven (DR 101, Universal, India).

\section{Determination of drug incorporation efficiency}

The drug incorporation efficiency of each formulation was determined by first extracting rifabutin from an accurately weighed quantity $(100 \mathrm{mg})$ of the beads using $0.01 \mathrm{M} \mathrm{HCl}(\mathrm{pH}$ 2.0) and determining the amount of the drug by high performance liquid chromatography (HPLC).

For HPLC determination of drug, the mobile phase consisted of $0.05 \mathrm{M}$ potassium dihydrogen phosphate and sodium acetate ( $\mathrm{pH}$ adjusted to 4.0 with acetic acid)/ acetonitrile $(53: 47, \mathrm{v} / \mathrm{v})$ at a flow rate of 1 $\mathrm{ml} / \mathrm{min}$ at $25{ }^{\circ} \mathrm{C}$. The HPLC system (Shimadzu, SPD-20A detector, LC-20AT pump) consisted of a UV detector set to 275 nm and Pursuit XRs C-8 analytical column (USP-L7). 
A standard solution of rifabutin $(100 \mu \mathrm{g} / \mathrm{ml})$ was prepared by weighing the appropriate amount of bulk rifabutin and dissolving it in the mobile phase. Further stock solutions were made by diluting the initial stock standard solution with mobile phase. A seven-point calibration curve ranging from 1 to $7 \mu \mathrm{g} / \mathrm{ml}$ was used for the quantification of rifabutin. A stock solution of $1.0 \mu \mathrm{g} / \mathrm{ml}$ was stored at $-30{ }^{\circ} \mathrm{C}$ and a sample of this stock solution was always injected together with the analyzed samples to verify the precision of the obtained concentrations of rifabutin in samples and controls from their peak area concentration response.

\section{Drug-polymer interaction}

Fourier Transform Infrared Spectroscopy (FTIR) was employed to study drug-polymer interactions. Spectra were recorded for the pure drug and drug-loaded beads using a FTIR facility (Shimadzu, model 8400S). The samples were prepared in $\mathrm{KBr}$ disks (2 mg sample in $200 \mathrm{mg} \mathrm{KBr}$ ). The scanning range was $400-4000 \mathrm{~cm}^{-1}$ and the resolution was 2 $\mathrm{cm}^{-1}$.

\section{Assessment of in vitro buoyancy of the beads}

In vitro study of bead bouyancy was performed using a USP dissolution apparatus type II (paddle type, Electrolab, Mumbai, India). The beads were dispersed in $500 \mathrm{ml}$ of $0.01 \mathrm{M} \mathrm{HCl}(\mathrm{pH} 2.0)$ at $37 \pm 1{ }^{0} \mathrm{C}$ with continuous agitation at $50 \mathrm{rpm}$ for $24 \mathrm{~h}$. The floating beads were separated from the submerged beads and their proportion (\%) was determined as described earlier $[3,4]$.

\section{Morphology and bead size analysis}

The size of the beads was determined with an optical microscope (Model $\mathrm{BH}-2$, Olympus, Japan) fitted with a stage and an ocular micrometer. Twenty dried beads were measured in order to determine the mean diameter of the beads. All measurements were in triplicate. The shape, surface morphology and internal structure of the dried beads were assessed with a scanning electron microscope (Leo 435VP, variable pressure, Oxford, U.K.) at various magnifications.

\section{In vitro drug release studies}

In vitro release of rifabutin from the beads was evaluated with a USP dissolution apparatus type II (paddle type, Electrolab, Mumbai, India) at $50 \mathrm{rpm}$. in $500 \mathrm{ml} 0.01 \mathrm{M}$ $\mathrm{HCl}(\mathrm{pH} 2.0)$ at $37 \pm 0.5{ }^{\circ} \mathrm{C}$, as per USP monograph for rifabutin capsules. At predetermined intervals, a 1-ml aliquot was withdrawn and replenished with an equal volume of fresh dissolution medium [5].The withdrawn samples were analyzed by HPLC as described earlier.

\section{Determination of mechanism of drug release}

The in vitro data were fitted to Higuchi's square root model [6] to analyze the kinetics of drug release from the beads (see Eq 1)

$\mathrm{Q}=\mathrm{K} \sqrt{ } \mathrm{t}$

where $Q$ is the amount of the drug released in time, $t, K$ is the release constant of the equation. The data were also subjected to Korsmeyer-Peppas power law[7,8] as in Eq 2.

$\mathrm{Mt} / \mathrm{M} \infty=\mathrm{Kt}^{\mathrm{n}}$

where $\mathrm{Mt} / \mathrm{M} \infty$ is the fraction of drug released in time, $\mathrm{t}, \mathrm{K}$ is the structural and geometric constant, and $\mathrm{n}$ is the release exponent.

\section{Statistical analysis}

All the data were analyzed by Student's t-test and one-way ANOVA to determine statistical difference in the results. A probability value of $p<0.05$ was considered statistically significant. The software used was SigmaPlot ${ }^{\circledR} 11$ (Systat Software Inc). 


\section{RESULTS}

\section{Morphological properties and size of beads}

The scanning electron micrographs (SEM) of rifabutin-loaded gellan beads are shown in Fig 1. The beads were spherical but exhibited a rough surface. The transverse section of the beads revealed a very porous interior. The mean bead diameter data, shown in Table 2, indicate that (at low calcium concentration) as the amount of gellan gum in the beads increased, diameter also increased $(p<0.05)$. However, there were no significant difference in bead size when the concentration of either calcium chloride or crosslinking agent was varied.

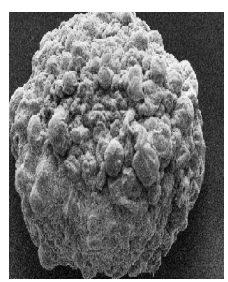

(a)

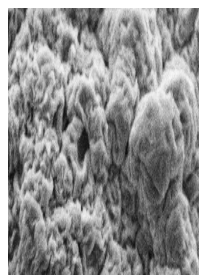

(b)

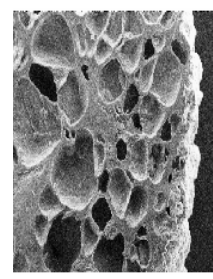

(c)
Figure 1: Scanning electron microscopy images of rifabutin-loaded bead: (a) shape (b) surface morphology (c) transverse section

Table 1: Composition of floating rifabutin beads

\begin{tabular}{ccc}
\hline $\begin{array}{c}\text { Batch } \\
\text { code }\end{array}$ & $\begin{array}{c}\text { Gellan gum } \\
(\% \mathbf{w} / \mathbf{v})\end{array}$ & $\mathbf{C a C l}_{\mathbf{2}}(\% \mathbf{w} / \mathbf{v})$ \\
\hline A1 & 1 & 3 \\
A2 & 1 & 4 \\
A3 & 1 & 5 \\
A4 & 2 & 3 \\
A5 & 2 & 4 \\
A6 & 2 & 5 \\
A7 & 3 & 3 \\
A8 & 3 & 4 \\
A9 & 3 & 5 \\
\hline
\end{tabular}

Each batch also contained 1.0 and $0.4 \%$ rifabutin and sodium bicarbonate, respectively)
Table 2: Some physiochemical characteristics of floating rifabutin beads

\begin{tabular}{ccccc}
\hline $\begin{array}{c}\text { Batch } \\
\text { code }\end{array}$ & $\begin{array}{c}\text { Diameter } \\
(\mathbf{m m})\end{array}$ & $\begin{array}{c}\text { Drug } \\
\text { Entrap- } \\
\text { ment (\%) }\end{array}$ & $\begin{array}{c}\text { Buoyancy } \\
(\%)\end{array}$ & $\mathbf{n}$ \\
\hline A1 & $1.26 \pm 0.04$ & $40.3 \pm 1.4$ & $98.8 \pm 1.8$ & 0.53 \\
A2 & $1.22 \pm 0.06$ & $44.7 \pm 1.4$ & $96.7 \pm 2.0$ & 0.54 \\
A3 & $1.20 \pm 0.05$ & $48.9 \pm 1.3$ & $94.7 \pm 1.9$ & 0.41 \\
A4 & $1.46 \pm 0.02$ & $46.1 \pm 1.2$ & $97.1 \pm 1.8$ & 0.41 \\
A5 & $1.42 \pm 0.05$ & $48.7 \pm 1.3$ & $95.3 \pm 2.1$ & 0.41 \\
A6 & $1.40 \pm 0.05$ & $53.9 \pm 1.3$ & $92.4 \pm 1.8$ & 0.42 \\
A7 & $1.52 \pm 0.03$ & $48.6 \pm 1.2$ & $95.8 \pm 1.7$ & 0.45 \\
A8 & $1.49 \pm 0.03$ & $53.9 \pm 1.3$ & $94.4 \pm 1.9$ & 0.46 \\
A9 & $1.48 \pm 0.02$ & $60.7 \pm 1.2$ & $90.2 \pm 1.7$ & 0.46 \\
\hline
\end{tabular}

\section{Drug entrapment (incorporation efficiency) of rifabutin beads}

Rifabutin entrapment ranged from 40.3 to $60.7 \%$ (see Table 2). Drug entrapment efficiency varied with polymer content and the calcium concentration of the gelation medium. Increase in calcium concentration enhanced drug encapsulation efficiency $(p<$ 0.05, compare batches A1 and A3; A4 and A6; and A7 and A9). Similarly, as the concentration of gellan gum rose, entrapment efficiency also increased (compare $A 1$ and A7; and A2 and A8). Overall, however, rifabutin-entrapment efficiency of the beads was low.

\section{Drug-polymer interaction}

The FTIR spectra of rifabutin, gellan gum, their physical mixtures and beads (not shown) indicate that there was no interaction between the drug and gellan.

\section{In vitro bead buoyancy}

As Table 2 shows, the floating properties of the formulations depended largely on sodium bicarbonate. While the control beads (without sodium bicarbonate) sank completely in the 
medium, the beads containing $0.4 \%$ $\mathrm{NaHCO}_{3}$ demonstrated good floating characteristics. There was no lag time as the beads floated immediately when placed in $0.01 \mathrm{M} \mathrm{HCl}$ and remained so for up to $18 \mathrm{~h}$.

\section{In vitro drug release properties}

Rifabutin release from four of the bead formulations in $0.01 \mathrm{M} \mathrm{HCl}(\mathrm{pH} 2.0)$ is shown in Fig 2. Release decreased $(p<0.05)$ with increase in calcium concentration and was characterized by an initial phase of rapid release (burst effect) due to the good solubility of the drug in the dissolution medium, followed by slow release of the drug. No significant difference $(p<0.05)$ in drug release was observed when gellan gum content was varied. Overall drug release from the beads was rapid, with $48-69 \%$ released within the first hour.

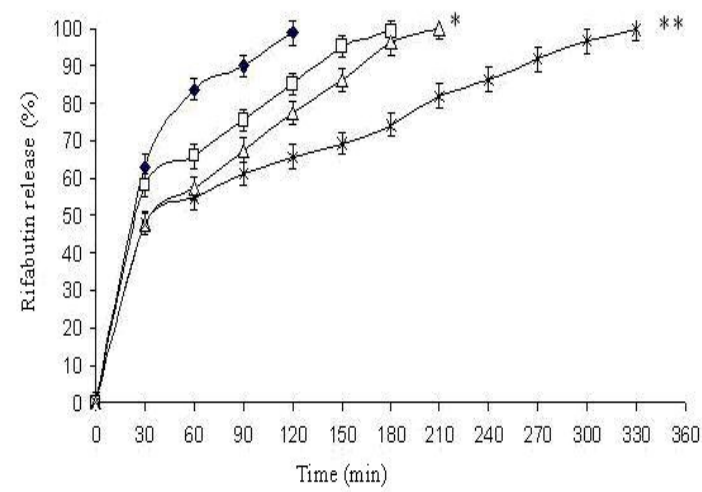

Figure 2: Cumulative rifabutin release from floating beads containing $2 \% / 3 \%$ gellan $/ \mathrm{CaCl}_{2}(\mathrm{~A} 4$, •), $2 \% / 5 \%$ gellan $/ \mathrm{CaCl}_{2} \quad(\mathrm{~A} 6, \quad \square), \quad 3 \% / 3 \%$ gellan $/ \mathrm{CaCl}_{2}(\mathrm{~A} 7, \Delta), 3 \% / 5 \%$ gellan $/ \mathrm{CaCl}_{2}(\mathrm{~A} 9, \mathrm{x})$. Note: ${ }^{*} p<0.05$ in comparison to $A 4$ and ${ }^{* *} p<$ 0.05 in comparison to $\mathrm{A} 6$.

\section{DISCUSSION}

The numerous pores in the bead interior may be directly related to the presence of sodium bicarbonate and is in agreement with the findings of Choi et al [4]. The increase in bead size with increasing gellan concentration could be attributed to increase in microviscosity of the polymeric dispersion, leading eventually to the formation of bigger beads [9].

The low drug entrapment efficiency of the beads may be due to the use of sodium bicarbonate as gas-generating agent, which on reacting with acetic acid, released carbon dioxide. The released carbon dioxide gets entrapped in the gel network, thus increasing bead porosity and decreasing the strength of the bead wall. As a result, the drug readily diffuses out into the gelation fluid. Stockwell and Davis [10] also attributed the low entrapment efficiency of alginate beads to violent gas generation.

Another reason for the low drug incorporation efficiency could be that metal cations exhibit a specific topological signature in the electron localization of their density interaction with ligands according to their "soft" or "hard" character". The degree of softness or hardness refers to the electron mobility or polarizability of a species. According to Pearson's Hard Soft Acid Base Principle [11] and Klopman's FMO (frontier molecular orbitals) analysis [12], hard acids tend to form strong bonds with hard bases to give chargecontrolled (ionic) complexes whereas soft acids tend to form strong bonds with soft bases to give FMO-controlled (covalent) complexes. In the present study, the hard base and hard acid are calcium ions and the $\mathrm{COO}^{-}$group on the gellan network, respectively. Therefore, interaction between calcium and the carboxylate group on the gellan molecule will be most preferred, but water is known to hamper hard acid-hard base interactions [13], being itself a hard base and therefore, competes with calcium ions. This could have hindered the approach of calcium ions to the $\mathrm{COO}^{-}$group, thus resulting in the formation of weak complexes that could not retain rifabutin adequately. Hence also, as the concentration of calcium in the gelation medium increased, the greater the amount of calcium ions available for reaction with $\mathrm{COO}^{-}$group; this might have resulted in strong aggregation of pairs of helices, leading to stronger gel structures that held more of the drug.

Trop J Pharm Res, February 2011;10 (1):65 
The increase in the drug entrapment efficiency of beads with increasing polymer concentration may be because increase in the gellan concentration resulted in the formation of larger beads which entrapped more drug. Furthermore, higher polymer concentration affords higher viscosity, which would hinder easy escape of the drug from the polymer matrix to the gelation medium.

Beads were floated in $0.01 \mathrm{M} \mathrm{HCl}$ for $18 \mathrm{~h}$. Upon contact with acid medium, sodium bicarbonate effervesces, releasing carbon dioxide. In this case, the released carbon dioxide was most likely entrapped in the bead gel network produced by the reaction of the calcium ions present in the gelation medium with gellan. The crosslinked threedimensional gel network would have restricted the outward diffusion of carbon dioxide, leading to an extended period of bouyancy.

\section{Rifabutin release}

The mechanism of crosslinking or gelation of gellan gum with calcium chloride is based on the formation of double helical junction zones followed by aggregation of double helical segments to form a three dimensional network by complexation with cations [14]. However, gellan [2] and alginate-based matrix drug delivery systems [15-17] often suffer from rapid drug release such as was observed in the present study. The reason for the rapid release may be explained by the fact that gelation and aggregation of gellan gum occur via chemical bonding between calcium and carboxylic groups in the gellan chains. Calcium, being a hard electrophile, interacts with the carboxylate group of gellan gum electrostatically. Such interactions tend to be readily hydrated and thus broken under aqueous conditions, resulting in rapid release of drug from the beads [18].

As the concentration of gellan gum increased, more carboxylate group side chains would be available for the formation of a stronger gellan-calcium network. However, this appeared not be the case in this study because increasing gellan concentration did not result in the expected reduction in the drug release of the beads. No significant difference $(p<0.05)$ in drug release was observed as gellan content was increased. This may be due to the inadequate availability of calcium ions for cross-linking with gellan in the beads.

\section{Drug release mechanism}

The range of correlation coefficient $\left(r^{2}\right)$ values for various release models applied to the release data were as follows: $0.7515-0.8737$ (zero order), $0.8954-0.8432$ ( $1^{\text {st }}$ order), and $0.9447-0.9926$ (Higuchi). This suggests that drug release from the beads predominantly followed Higuchi model as the $r^{2}$ values were higher than for other models. When the invitro drug release data were fitted to the exponential equation $\mathrm{M}_{\mathrm{t}} / \mathrm{M}_{\infty}=\mathrm{Kt}^{\mathrm{n}}$, it was found that drug release also followed an anomalous non-Fickian diffusion pattern, as indicated by the release exponent values $(n)$ which were in the range $0.41-0.53$.

\section{CONCLUSION}

Since the incorporation efficiency and drug release could be controlled by the regulation of the process factors, floating gellan gum beads can be further developed for use as a oral stomach-specific release system for the treatment of stomach-specific infections such as multi-drug resistant Helicobacter Pylori infection.

\section{ACKNOWLEDGEMENT}

The authors are thankful to Dr RM Dubey, Managing Director of IFTM, Moradabad, India for providing facilities and institutional research grant for the work.

\section{CONFLICT OF INTERESTS}

The authors declare no conflict of interest.

Trop J Pharm Res, February 2011;10 (1):66 


\section{REFERENCES}

1. Chandrasekaran $R$, Radha $A$, Thailambal VG. Roles of potassium ions acetyl and L-glyceryl groups in native gellan double helix: An X-ray study. Carbohydr Polym. 1992; 224: 1-17.

2. Singh BN, Kim KH. Effects of divalent cations on drug encapsulation efficiency of deacylated gellan gum. J Microencapsul. 2005; 22: 761771.

3. Kanesaka S, Watanabe T, Matsukawa S. Binding effect of $\mathrm{Cu}^{2+}$ as a trigger on the sol-to-gel and the coil-to-helix transition processes of polysaccharide, gellan gum. Biomacromolecules. 2004; 5: 863-68.

4. Choi BY, Park HJ, Hwang SJ, Park JB. Preparation of alginate beads for floating drug delivery: effects of $\mathrm{CO}_{2}$ gas forming agents. Int $J$ Pharm. 2002; 239: 81-91.

5. United State Pharmacopeia 31/National Formulary 26, Vol 3. USP Convention, Rockville, MD 20852. Asian Edition, 2008; pp 3181-3182.

6. Higuchi T. Mechanism of sustained-action medication: theoretical analysis of rate of release of solid drugs dispersed in solid matrices. J Pharm Sci. 1963; 52: 1145-1149.

7. Korsmeyer R, Gurny R, Peppas N. Mechanisms of solute release from porous hydrophilic polymers. Int J Pharm. 1983; 15: 25-35.

8. Peppas NA. Analysis of Fickian and non-Fickian drug release from polymers. Pharm Acta Helv. 1985; 60: 110-111.

9. Rajnikanth PS, Mishra B. Stomach-Site Specific Drug Delivery System of Clarithromycin for Eradication of Helicobacter pylori. Chem Pharm Bull. 2009; 57(10): 1068-1075.
10. Stockwell AF, Davis SS. In vitro evaluation of alginate gel system as sustained release drug delivery systems, J Control Release.1986; 3: 167-175.

11. Dipankar D. On Pearson's HSAB principle. Inorg Chem. 1992; 31(13): 2797-2800.

12. Fernando $M$, Renato RC, Arie JA. Introduction of external field effects in the frontier molecular orbital theory of chemical reactivity. Int J Quant Chem. 2004; 44(S26): $751-760$.

13. Engberts JBFN, Feringa BL, Erik $K$, Sijbren $O$. Lewis-acid catalysis of carbon- carbon bond forming reactions in water. Recl Trav Chim. 1996; 115: 457-464.

14. Brahma NS, Kwon HK. Effects of divalent cations on drug encapsulation efficiency of deacylated gellan gum. J Microencapsul. 2005; 22(7): 761-771.

15. Aslani $P$, Kennedy RA. Effect of gelation conditions and dissolution media on the release of paracetamol from alginate gel beads. $J$ Microencapsul. 1996; 13: 601-614.

16. Kikuchi A, Kawabuchi M, Sugihara M, Sakurai $Y$, Okano T. Pulsed dextran release from calcium-alginate gel beads. $J$ Control Release. 1997; 47: 21-29.

17. Ostberg T, Lund EM, Graffner C. Calcium alginate matrixes for oral multiple unit administration: IV. Release characteristics in different media. Int J Pharm. 1994; 112: 241-248.

18. Mutasem OT, Wissam $N$, Adel A, Hatim SA. Sodium lauryl sulfate impedes drug release from zinc-crosslinked alginate beads: Switching from enteric coating release into biphasic profiles. Int J Pharm. 2008; 350: 291300. 